\title{
Fuentes de contaminación intradomiciliaria y enfermedad respiratoria en jardines infantiles y salas cunas de Temuco y Padre Las Casas, Chile
}

\author{
Edith Rivas $\mathbf{R}^{\mathbf{l a}}$, Sara Barrios $\mathbf{C}^{\mathbf{l b}}$, Anita Dorner Plc, \\ Ximena $\mathbf{O}$ sorio $\mathrm{S}^{1 \mathrm{~d}}$. \\ Association between indoor \\ contamination and respiratory diseases \\ in children living in Temuco \\ and Padre Las Casas, Chile
}

Background: Indoor air pollution, is the main cause of population exposure to polluting agents. Aim: To establish an environmental profile of indoor contamination emission sources in families of children under 5 years that assist to kindergartens in Temuco and Padre Las Casas. To associate respiratory disease episodes in children with indoor contamination. Material and methods: Cross sectional analysis of 355 family groups subjected to questionnaires about indoor contamination and number of respiratory disease episodes. Results: Forty six percent of mothers or caregivers smoked, 37\% smoked at home and 93\% smoked one to two cigarettes per day. There was a significant association between respiratory diseases in children and drying clothes in the kitchen, using firewood for heating and the presence of humidity in the dwelling. Mothers identified as indoor contaminants the use of braziers in $76 \%$ of cases and firewood stoves in $24 \%$. Ninety seven percent considered that these appliances were detrimental for respiratory health. Conclusions: The lack of awareness about indoor contamination among subjects of low socioeconomic status, should prompt educational campaigns to modify behaviors in their dwellings (Rev Méd Chile 2008; 136: 767-74).

(Key words: Air pollution, indoor; Infants; Respiratory tract diseases)

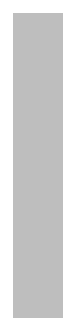

\footnotetext{
Recibido el 6 de junio, 2007. Aceptado el 24 de marzo, 2008.

Financiado por la Dirección de Investigación de la Universidad de La Frontera. Proyecto de investigación № 120406.

${ }^{1}$ Departamento de Pediatría y Cirugía Infantil, Facultad de Medicina, Universidad de La Frontera. Temuco, Chile.

aEnfermera Magíster en Salud Pública. Doctor en Enfermería.

bEnfermera Magíster en Educación. Doctor en Enfermería.

'Enfermera en Calidad en Sistemas de Gestión de Calidad

dEnfermera Magíster en Enfermería.
}

Correspondencia a: Edith Rivas Riveros. Departamento de Pediatría y Cirugía Infantil, Facultad de Medicina, Universidad de La Frontera. Casilla 54-D, Temuco, Chile. Telefono: (56-45) 325769 Fax: (56-45) 325710. E mail: erivas@ufro.cl. 
L a a contaminación de interiores representa un riesgo para la población, por sus efectos sobre la salud, situación que se acentúa por la permanencia de los individuos en ambientes interiores (80\%-90\% del tiempo) y la deficiente ventilación en viviendas y lugares de trabajo ${ }^{1-3}$. Conjuntamente, las condiciones climáticas en la Región de La Araucanía, hacen indispensable el uso de sistemas de calefacción -esencialmente a leñadado su bajo costo, acceso y tradición ${ }^{4}$.

Las consecuencias de los contaminantes sobre la salud son variables y dependen en gran medida de: tipo de contaminante, concentración, tiempo de exposición y de las reacciones con otros contaminantes para formar sustancias más tóxicas $^{1}$. Al respecto, se señala que la contaminación depende de: a) la emisión, debida al hacinamiento, presencia de animales, tabaquismo y uso de combustibles y otros productos domésticos; b) intercambio de aire con el exterior determinado por ventilación, aislación térmica e infiltración; c) eliminación de contaminantes por filtros de aire y adsorción; y d) dilución de contaminantes, dependiente del volumen de aire de las habitaciones y, por ende, del diseño de la construcción ${ }^{2}$.

Los efectos de la contaminación, también se pueden expresar diferidos en el tiempo, tales como mutaciones, malformaciones congénitas y en especial alteraciones de la programación celular (imprinting hormonal), estos últimos después de una exposición durante el periodo perinatal o infantil precoz y que han sido propuestos como favorecedores del desarrollo $2,5-6$.

Algunas investigaciones han demostrado que el uso de carbón, leña y parafina como combustibles, incrementan la prevalencia de enfermedades respiratorias ${ }^{7-8}$. Estos combustibles son más económicos que el gas y la electricidad y son usados con mayor frecuencia en zonas urbanas y rurales de extrema pobreza ${ }^{1}$.

La investigación, trata de determinar ¿cuáles son las fuentes emisoras de contaminación intradomiciliaria y cuál es su relación con la presencia de enfermedad respiratoria en niños menores de 5 años que asisten a los jardines infantiles de las comunas de Temuco y Padre Las Casas?

Efectos sobre la salud. Los efectos sobre la salud se dividen en: a) agudos, como irritación de ojos y nariz, aumento de las infecciones respiratorias agudas altas y bajas, exacerbaciones del asma bronquial, de enfermedad pulmonar obstructiva crónica, de cardiopatías y aumento de las tasas de mortalidad $^{9-10}$ y b) efectos crónicos o diferidos, como el cáncer pulmonar ${ }^{11}$. En estudios de asociación entre contaminación y morbilidad respiratoria, se ha argumentado que la relación patogénica debería relacionarse preferentemente con el ambiente más próximo de cada sujeto. Por ello, se han realizado mediciones de contaminación intramuros y han encontrado valores más altos de $\mathrm{PM}_{10}$ que los de ambientes exteriores ${ }^{12}$, por lo que la contaminación medida por monitores ambientales a lo menos minimiza la exposición real a la que está sometido el sujeto.

El humo de tabaco es el principal componente de la contaminación intradomiciliaria ${ }^{2}$, evidenciándose que la exposición pasiva a este contaminante produce irritación ocular, cefalea, irritación nasal y tos. En niños asmáticos la exposición al humo de tabaco ambiental puede exacerbar sus síntomas $^{13}$ y producir deterioro significativo de la función pulmonar, condicionando la aparición de cuadros respiratorios de mayor gravedad ${ }^{14}$.

En población infantil, la exposición involuntaria al humo de cigarrillo aumenta la frecuencia de infecciones respiratorias bajas y de síntomas respiratorios, reduce el nivel esperado de $\mathrm{VEF}_{1} \mathrm{y} \mathrm{FEF}_{25}$ $75^{15}$ y aumenta el riesgo de desarrollar otitis media $^{16}$. La exposición al humo de leña puede aumentar la frecuencia de infecciones respiratorias bajas ${ }^{17}$. Estudios en lactantes menores demostraron que la exposición a humo de cigarrillo, determinó un aumento significativo de las concentraciones de cotinina en saliva y orina; además hubo una relación directa entre el número de cigarrillos fumados por la madre, en las $24 \mathrm{~h}$ previas, y las concentraciones de cotinina alcanzados. En escolares se ha demostrado también la relación entre la concentración y el hábito tabáquico, siendo más determinante el materno ${ }^{18}$.

Por otra parte, investigaciones han demostrado que el uso de carbón, leña y parafina como combustibles, incrementa la prevalencia de enfermedades respiratorias ${ }^{7}$. Estos combustibles son más económicos que el gas y la electricidad y son usados con mayor frecuencia en zonas urbanas y rurales de extrema pobreza. 


\section{MATERIAL Y MÉTODO}

Diseño descriptivo, de corte transversal, constituido por muestra de 355 grupos familiares, calculada con una probabilidad de $95 \%$ y margen de error de 5\%. La recopilación de la información se realizó en dos cuestionarios: (MCI) medición de la contaminación intradomiciliaria y (MEER) medición de episodios de enfermedad respiratoria (bronquitis aguda, bronquiolitis, resfrío común y asma bronquial).

En los cuestionarios se utilizó validez de fachada (mide que esté bien construido el instrumento, opinión emitida por el experto). Para variables cualitativas se presentan frecuencias absolutas y relativas (porcentajes); los datos cuantitativos, en media y desviación estándar. La relación entre las variables cualitativas se efectuó a través de la prueba de chi cuadrado (test exacto de Fisher para tablas de 2x2). El nivel de significación establecido fue de $5 \%$. Los datos fueron procesados y analizados con el paquete estadístico Stata 8.0.

\section{RESUlTADOS}

La investigación planteó los resultados desde la situación de salud de los menores, la medición del hábito tabáquico en las madres y la relación entre enfermedad respiratoria y conductas de aseo en el hogar, combustibles para calefacción, hábito tabáquico y presencia de agentes biológicos. Conjuntamente se realizó evaluación cognitiva respecto de contaminación intradomiciliaria.

Se encuestó a un total de 355 madres de niños que asistieron a jardines infantiles y salas cunas, la edad promedio de los niños fue de 3 años (DS: 1,34), la edad promedio de las madres fue de 29 años (DS: 7,02). Los grupos familiares estaban constituidos por un promedio de 2,7 adultos (DS: 1,25) y 1,96 niños (DS: 0,889).

El $24,1 \%$ no contaba con sistema previsional y $57,4 \%$ se encontraba adscrito a FONASA. En 79,6\% el cuidador era la madre y en $17,7 \%$ abuelos u otros parientes. El 82,0\% contaba con vivienda de madera, en $57,4 \%$ el piso de su vivienda era de madera y $14,6 \%$ cubrepiso (alfombra de $<3 \mathrm{~mm}$ de grosor). El promedio de dormitorios fue de 2,35 (DS 1,03), y camas 3,34 (DS 1,39). El 96,3\% de las viviendas presentaba alcantarillado y $97,6 \%$ agua potable, en el entorno de la vivienda 91\% corresponde a calles pavimentadas.

En cuanto, a la presencia de enfermedades respiratorias, consultas y hospitalizaciones, $41,3 \%$ presenta 2-3 episodios respiratorios, $43,1 \%$ presenta 0-1 consultas médicas y $94,4 \%$ no ha presentado hospitalizaciones.

En hábitos de aseo, 96,3\% realiza aseo 4 a 7 veces a la semana, $79,4 \%$ extrae el polvo por barrido, no aspira y no trapea, 35,9\% realiza aseo de muebles con paño seco y $64,1 \%$ con paño húmedo. El 90,5\% seca la ropa fuera de la casa y el 15,6\% dentro de la casa (dormitorio, cocina, comedor). En ventilación de la casa: 87,6\% abre las ventanas diariamente. En contaminantes biológicos presentes en el domicilio: $32,3 \%$ la madre informa presencia de ácaros, $24,6 \%$ hongos y $43,1 \%$ moscas.

En medición de hábito tabáquico, 46,3\% de las madres y cuidadoras lo presentan, $32,0 \%$ reconoce a los amigos como la influencia para la condición de fumador, 36,8\% fuma en el domicilio, 93,1\% fuma 1-2 cigarrillos al día. La edad de inicio de hábito es 16,88 media y DS 4,172 (Tabla 1). Se demostró una asociación estadísticamente significativa entre enfermedad respiratoria en el menor y conducta secado de ropa en la cocina ( $p: 0,04)$ (Tabla 2); uso de leña para calefacción (p: 0,05) (Tabla 3) y presencia de hongos y humedad en el hogar (p: 0,04$)$, la conducta extracción de polvo por trapeo húmedo presentó una asociación de $\mathrm{p}$ : 0,079 (Tabla 4).

\section{Tabla 1. Medición de hábito tabáquico en madres y cuidadoras de lactantes y preescolares, asistentes a jardines infantiles y salas cunas, Temuco y Padre Las C asas, 2004.}

\begin{tabular}{|llr|}
\hline & & $\%$ \\
\hline Hábito tabáquico & & 46,3 \\
Influencia - condición fumador & Amigos & 32,0 \\
& Sin influencia & 50,2 \\
Lugar físico donde fuma & Trabajo & 27,5 \\
& Domicilio & 36,8 \\
Cantidad que fuma al día & $1-2$ & 93,1 \\
& $3-4$ & 6,9 \\
\hline
\end{tabular}

Edad de inicio de hábito: Media 16,88ะ4,172 DS. 
Tabla 2. Relación entre conductas de aseo en el hogar y enfermedad respiratoria, en madres y cuidadoras de niños asistentes a jardines infantiles y salas cunas, Temuco y Padre Las Casas, 2004

\begin{tabular}{|llrrr|}
\hline Conducta & & Con enf. & Sin enf & $\mathrm{p}$ \\
\hline \multirow{2}{*}{ Frecuencia aseo-hogar } & 4 a 7 v/semana & 94,9 & 96,7 & 0,32 \\
& 1 vez a la semana & 5,1 & 3,3 & \\
Extracción de polvo & Barre & 83,3 & 78,3 & 0,20 \\
& Aspira & 39,7 & 40,6 & 0,49 \\
Aseo muebles & Trapea & 44,8 & 46,6 & 0,43 \\
Secado de ropa & Paño húmedo & 43,5 & 34,0 & 0,19 \\
& Paño seco & 56,5 & 66,0 & \\
& Patio & 89,7 & 90,6 & 0,47 \\
Ventilación-casa & Cocina & 42,3 & 31,0 & 0,04 \\
Combustible-cocinar & Comedor & 8,9 & 9,0 & 0,59 \\
& Dormitorio & 6,0 & 4,0 & 0,26 \\
& Diario & 11,5 & 12,6 & 0,48 \\
& Leña & 34,6 & 30,0 & 0,25 \\
\hline
\end{tabular}

Tabla 3. Relación combustibles para cal efacción y presencia de enfermedad respiratoria en niños asistentes a jardines infantiles y salas cunas, Temuco y Padre Las C asas, 2004

\begin{tabular}{|lccc|}
\hline & Con enf & Sin enf & $\mathrm{p}$ \\
\hline Calefacción - carbón & 2,6 & 1,7 & 0,442 \\
Calefacción - leña & 32,8 & 21,9 & 0,050 \\
Calefacción - gas & 91,4 & 89,1 & 0,347 \\
Calefacción - parafina & 1,3 & 1,0 & 0,605 \\
Calefacción - electricidad & 1,0 & 1,3 & 0,605 \\
\hline
\end{tabular}

Tabla 4. Relación entre la conducta de aseo del hogar y presencia de enfermedad respiratoria en niños asistentes a jardines infantiles y salas cunas, Temuco y Padre Las C asas, 2004

\begin{tabular}{|lccc|}
\hline & Con enf & Sin enf & $\mathrm{p}$ \\
\hline Frecuencia aseo correcta & 95,9 & 98,4 & 0,219 \\
Frecuencia ventilación correcta & 12,4 & 12,5 & 0,562 \\
Extracción polvo por barrido & 78,3 & 84,4 & 0,180 \\
Extracción polvo aspirando & 41,4 & 35,9 & 0,252 \\
Extracción polvo por trapeo & 48,1 & 37,5 & 0,079 \\
Aseo muebles con paño húmedo & 64,0 & 64,1 & 0,813 \\
Secado ropas exterior de la casa & 91,1 & 87,5 & 0,248 \\
\hline
\end{tabular}


Tabla 5. Evaluación cognitiva respecto de contaminación ambiental en madres de lactantes y preescolares que asisten a jardines infantiles y salas cunas, Temuco y Padre Las Casas, 2004

\begin{tabular}{|llc|}
\hline Evaluación cognitiva & & $\%$ \\
\hline Calefactor más contaminante & Brasero & 75,7 \\
& Cocina leña & 24,3 \\
Daño en la salud & Respiratorio & 97,4 \\
Contaminantes intradomiciliarios & Carbón & 65,3 \\
& Leña & 25,1 \\
& Polvo & 62,2 \\
& Humedad & 50,8 \\
& Parafina & 54,2 \\
Contaminantes extradomiciliarios & Cigarro & 74,6 \\
& Humo & 89,4 \\
& Combustión de vehículo & 74,6 \\
& Tierra-polvo & 46,8 \\
& Combustión de fábrica & 71,5 \\
\hline
\end{tabular}

Evaluación cognitiva. Dentro de los contaminantes intradomiciliarios y calefactores más contaminantes, las madres identifican en $75,7 \%$ al brasero, $24,3 \%$ a la cocina de leña y $97,4 \%$ identifica estos elementos que producen daño respiratorio en la salud (Tabla 5).

Como contaminantes intradomiciliarios $74,6 \%$ identifican al cigarrillo, 65,3\% carbón, 62,2\% polvo y $54,2 \%$ parafina. En los contaminantes extradomiciliarios: 89,4\% humo, 74,6\% combustión de vehículo, 46,8\% tierra-polvo y 71,5\% combustión de fábrica (Tabla 5).

\section{DisCUSIÓN}

Los resultados muestran mayores cifras de hábito tabáquico en madres y cuidadoras $(46,3 \%)$ y asociación estadísticamente significativa entre conductas de aseo en el hogar y enfermedad respiratoria en el menor, secado de ropa en la cocina (p: 0,04); extracción de polvo por trapeo húmedo (p: 0,079); calefacción a leña (p: 0,05) y presencia de humedad en el hogar (p: 0,04).

Las cifras de hábito tabáquico en madres y cuidadoras encontradas en nuestro estudio $(46,3 \%)$ son levemente mayores a estudios de Medina y Kaempffer; quienes observaron un incremento considerable de la prevalencia del hábito tabáquico en mujeres entre los años 1971 y 1984 (24\% a $39 \%)^{14}$; Gutiérrez et $\mathrm{al}^{19}$ prevalencia de $35 \%$ (en mujeres fumadoras con edad promedio de 32,6 años) y Osorio et al, prevalencia de 37,9\% (mujeres, enfermeras en la IX Región). En este último estudio la edad promedio de las madres fue de 29,4 años de edad, situación que podría influir en el aumento de las cifras ${ }^{20}$.

La relación entre conducta de aseo y presencia de enfermedad respiratoria en el menor, demostrada a través de la asociación estadísticamente significativa entre secado de ropa en la cocina ( $p$ : $0,04)$; extracción de polvo por trapeo húmedo ( $\mathrm{p}$ : 0,079); calefacción a leña ( $\mathrm{p}: 0,05)$ y presencia de humedad en el hogar (p: 0,04) es también señalada en estudios de Burgos y Saldías ${ }^{21}$, Cifuentes $^{18}$, González y Lisboa ${ }^{22}$ quienes refieren que las IRA presentan un comportamiento anual que tiende a concentrarse en los meses de invierno, situación que se relaciona directamente con el uso de sistemas de calefacción y presencia de humedad en el hogar. Frente a estos resultados es importante considerar el elevado porcentaje de tiempo, que pasan los niños en la cocina, condicionado, por la mayor temperatura del recinto y foco de actividades del hogar, en las familias de nuestra región y la importancia que adquiere la presencia 
de humedad en las viviendas. Además se debe recordar que la ventilación está limitada en los hogares, dada las copiosas lluvias y vientos, lo que contribuiría a la concentración de virus respiratorios.

No se exploró asociación entre secado de ropa en otras habitaciones, debido a que la pregunta se realizó abierta y las respuestas correspondieron a cocina y exterior del hogar. Por otra parte la contaminación atmosférica en la región es un problema que se ha agravado estos últimos años. Al respecto un estudio de Barrios et al, encontraron relación entre la contaminación atmosférica $\mathrm{PM}_{10} \mathrm{y}$ el aumento en el número de atenciones de IRA (SBO) en menores de 5 años ${ }^{23}$; RomeroPlaceres et $\mathrm{al}^{24}$ mostraron que aún los bajos niveles de contaminación del aire en las ciudades son dañinos para el tracto respiratorio de los menores de edad y que ello se relaciona con el aumento del riesgo de padecer IRA.

Zamorano el $\mathrm{at}^{25}$, no observaron ninguna asociación entre contaminación atmosférica, situación climática y número de consultas.

Rivas $^{26}$, encontró asociación entre síndrome bronquial obstructivo, etnia y conducta de cuidado, las madres revelaron déficit en la conducta de cuidado hacia su hijo (conducta de calefaccionar, ventilar y evitar fuentes de contagio en el hogar). Sanhueza et al, en un estudio en la región de la Araucanía, mostraron que los sitios urbanos de Temuco y Padre Las Casas presentan contaminación atmosférica de material particulado $\mathrm{PM}_{10} \mathrm{y}$ una fuerte relación de mortalidad diaria con dichos niveles de contaminación, principalmente por las emisiones generadas a partir de la combustión de leña, proveniente de actividades domésticas e industriales ${ }^{27}$.

En la evaluación cognitiva, las madres reconocieron en $97,4 \%$ que los calefactores como brasero y cocina a leña producen daño respiratorio en la salud y como calefactor de mayor contaminación al brasero con $75,7 \%$. Conjuntamente la situación de las enfermedades respiratorias en los niños en la región, se ve además agravada por la condición de vulnerabilidad social (pobreza y etnia mapuche).
En esta situación de contaminación intradomiciliaria y enfermedad respiratoria, los resultados sugieren que los niveles de contaminantes atmosféricos en la cuidad de Temuco afectan la salud respiratoria de los niños, por lo que se requiere de medidas de control. Al respecto diversos autores señalan, la necesidad de introducir estrategias de mitigación, planes de descontaminación o propuestas de educación ambiental como respuestas que la sociedad demanda para vivir en un ambiente sostenible ${ }^{28-32}$. Las medidas de control de la contaminación del aire en ambientes contemplan: mejorar la ventilación, eliminar la fuente, modificar la fuente, purificar el aire y promover cambios conductuales en las personas ${ }^{33}$.

Las medidas específicas, dependerán del contaminante que se pretende controlar, por ejemplo en el humo de tabaco, se debe eliminar la fuente; otras medidas es utilizar energía más limpia.

El aporte del estudio se traduce en nueva evidencia, para la introducción de medidas de control frente a la problemática local de contaminación y enfermedad respiratoria en los niños, evidenciado por la mayor prevalencia de hábito tabáquico en madres y cuidadoras $(46,3 \%)$ y la asociación entre conductas de aseo en el hogar y enfermedad respiratoria. Se recomienda continuar con el desarrollo de programas educativos en educación ambiental, que incorpore al niño, familia y comunidad (colegios, jardines infantiles), debido a que el colegio se convierte en un ente motivador para el cambio de conductas. Conjuntamente se reafirma la necesidad de incentivar el mensaje educativo a través de educación individual en consejerías a madres de niños con factores de riesgo para IRA, y reforzar conductas en el hogar (manejo aseo y humedad) y fortalecer la propuesta educativa, desarrollando conciencia, conocimientos, actitudes y la participación de los padres y educadores.

Se concluye que la prevalencia de tabaquismo en las madres, a nivel local es mayor a la del país y la existencia de asociación entre la humedad en el hogar y enfermedad respiratoria. 


\section{REFERENCIAS}

1. Cáceres A, Adonis M, Retamal C, Ancic P, Valencia $\mathrm{M}$ ET AL. Contaminación intradomiciliaria en un sector de extrema pobreza de la comuna de La Pintana. Rev Méd Chile 2001; 129, 1: 33-42.

2. Samet J, Marbury C, Spengler J. Health effects and sources of indoor air pollution (Part $1 \& 2$ ). Am Rev Respir Dis 1987; 136: 1486-508 y 137: 221-42.

3. Boleij D, BruneKreef E. Association between radical generation by urban particles and lung function in schoolchildren. J Toxicol Environ Health A 2006; 69, 22: 2007-8.

4. Servicio de Salud del Medio Ambiente. 2005. Información general. Contaminación puertas adentro. Disponible en: http//sesma.cl/sitio/pag/ aire/Indexjs3aire007.asp [Consultado el 25 de julio de 2006].

5. TChernitchin AN, TChernitchin NN, Mena MA, Unda C, Sото J. Imprinting: Perinatal exposures cause the development of during the adult age. Acta Biol Hung 1999; 50: 425-40.

6. TCHERNITCHIN AN. Perinatal exposure to chemical agents: delayed effects by the mechanism of imprinting (cell programming). ARBS Ann Rev Biomed Sci 2005; 7: 68-126.

7. LESLE GB, HaRAPRASAD V. Indoor air pollution from combustion sources in developing countries. Indoor Environ 1993; 2: 4-13.

8. Raiyani CV, Jani JP, Desai NM, Shah SH, Kashyap SK. Assessment of indoor exposure to polycyclic aromatic hydrocarbons for urban poor using various types of cooking fuels. Bull Environ Contam Toxicol 1993; 50: 757-63.

9. Ostro B, Sánchez JM, Aranda C, Eskeland GS. Air pollution and mortality: results from a study of Santiago, Chile. J Expo Anal Environ Epidemiol 1996; 6: 97-114.

10. Román O, Mancila P, Prieto MJ. Contaminación atmosférica y daño cardiovascular. Rev Méd Chile 2004; 132: 761-7.

11. Rosales JA, Torres VM, Olaiz G, Borja VH. Los efectos agudos de la contaminación del aire en la salud de la población: Evidencias de estudios epidemiológicos. Salud Pública Méx 2001; 43: 544-55.

12. Cáceres L, Adonis M, Retamal $C$, Ancic P, Vaiencia M ET AL. Contaminación intradomiciliaria en un sector de extrema pobreza de la comuna de La Pintana. Rev Méd Chile 2001; 129: 33-42.
13. EsAMAI FO. Relationship between exposure to tobacco smoke and bronchial asthma in children: a review. East Afr Med J 1998; 75: 47-50.

14. Medina E, KaempFFer A. Tabaquismo y salud en Chile. Bol of Sanit Panam 1991; 111, 2: 112-20.

15. Tager JB, Weiss ST, Munoz A, Rosner B, Speizer FE. Longitudinal study of the effects of maternal smoking on pulmonary function in children. $\mathrm{N}$ Engl J Med 1983; 309: 699-703.

16. Pukander J, Luotonen J, Timonen M, Karmer P. Factors affecting the ocurrence of acute otitis media among 2-3-year-old urban children. Acta Otolaryngol 1985; 100: 260-5.

17. OyaRzúN M. Factores ambientales relacionados con la gravedad del asma. Rev Chil Enferm Respir 2004; 20, 1: 25-9.

18. CifuENTES L 2001. Infecciones respiratorias agudas en pediatría ambulatoria. Publicaciones Pontificia Universidad Católica de Chile. Escuela de Medicina. Disponible en: http://escuela.med.puc.cl/pag in as / p ublicacion es / Manual Ped/ InfecRespAghtml [Consultado el 30 de octubre de 2006].

19. Gutiérrez M, Rioseco F, Rojas A, Casanova D, Cordero M, Schiaffino M. Prevalencia de tabaquismo en la población general de Valparaíso y Viña del Mar. Rev Méd Chile 1995; 123: 250-6.

20. Osorio X, RIvas E, Jara J. Prevalencia de tabaquismo en enfermeras de la IX Región, Chile. Rev Méd Chile 2003; 131, 3: 269-74.

21. Burgos P, Saldías F. 2001. Neumonía adquirida en la comunidad (NAC). Publicaciones Pontificia Universidad Católica de Chile. Escuela de Medicina. Disponible en: http:/ escuela.med.puc.cl/paginas/publicaciones/TemasMedicinaInterna/ nac.html (Consultado el 8 de noviembre de 2006).

22. GonZÁLEZ J, LsBoA C. 2001. Enfermedad bronquial obstructiva crónica. Publicaciones Pontificia Universidad Católica de Chile. Escuela de Medicina. Disponible en: http:/ escuela.med.puc.cl/paginas/ publicaciones/TemasMedicinaInterna/ epoc.html (Consultado el 8 de noviembre de 2006(.

23. Barrios S, Pena-Cortés F, Osses S. Efectos de la contaminación atmosférica por material particulado en las enfermedades respiratorias agudas en menores de 5 años. Cienc Enferm 2004; 10, 2: 21-9.

24. Romero-Placeres M, Mas-Bermejo P, Lacasaña-Navarro M, Téllez M, Aguilar-Valdés J et al. Air 
pollution, bronchial asthma, and acute respirator and infections in children less years of age, Habana City. Salud Pública Méx 2004; 46, 3: 222-33.

25. Zamorano A, Márquez S, Aránguiz JL, Bedregal P, SánCHEZ I. Association of acute bronchiolitis with climate factors and environmental contamination. Rev Méd Chil 2003; 131, 10: 1117-22.

26. RIVAS E. 2006. Factores asociados a síndrome bronquial obstructivo y neumonía en el lactante menor de un año con un enfoque de cuidado. Comunicación Personal. Programa de Doctorado en Enfermería. Universidad Nacional de Tucumán.

27. Sanhueza P, Vargas C, Meliado P. Impacto de la contaminación del aire por PM10 sobre la mortalidad diaria en Temuco. Rev Méd Chile 2006; 134: 754-61.
28. Oyarzún M. Contaminación atmosférica de Santiago: Realidades y proyecciones futuras. Rev Chil Enf Respir 1991; 7: 183-4.

29. OlaEta I. Métodos de monitoreo de la calidad del aire en la Región Metropolitana (Red MACAM). Rev Chil Enf Respir 1991; 7: 186-90.

30. Belo S, Oyarzún M. Efectos adversos para la salud de los contaminantes atmosféricos. Rev Chil Enf Respir 1991; 7: 198-205.

31. BARRUETO L. 2002. Enfermedades Respiratorias por Contaminación Atmosférica en Santiago. Disponible en: http://www.usach.cl/ima (Consultado el 10 de marzo de 2006.

32. Prendez M, Ortiz J, Zolezzi S, Campos C, Apablaza N. Aerosoles atmosféricos de naturaleza inorgánica. Contaminación en Santiago de Chile. Rev Chil Enf Respir 1991; 7: 224-37.

33. Spengler J, SeXton K. Indoor air pollution: a public health perspective. Science 1983; 221: 9-17. 